\title{
Evidence for nanocoulomb charges on spider ballooning silk
}

\author{
E. L. Morley ${ }^{1, *}$ and P. W. Gorham $\odot^{2, \dagger}$ \\ ${ }^{1}$ School of Biological Sciences, University of Bristol, 24 Tyndall Avenue, Bristol BS8 1TQ, United Kingdom \\ ${ }^{2}$ Department of Physics \& Astronomy, University of Hawaii at Manoa, 2505 Correa Rd., Honolulu, Hawaii 96822, USA
}

(Received 10 December 2019; revised 5 March 2020; accepted 6 March 2020; published 9 July 2020)

\begin{abstract}
We report on three launches of ballooning Erigone spiders observed in a $0.9 \mathrm{~m}^{3}$ laboratory chamber, controlled under conditions where no significant air motion was possible. These launches were elicited by vertical, downward-oriented electric fields within the chamber, and the motions indicate clearly that negative electric charge on the ballooning silk, subject to the Coulomb force, produced the lift observed in each launch. We estimate the total charge required under plausible assumptions, and find that at least $1.15 \mathrm{nC}$ is necessary in each case. The charge is likely to be nonuniformly distributed, favoring initial longitudinal mobility of electrons along the fresh silk during extrusion. These results demonstrate that spiders are able to utilize charge on their silk to attain electrostatic flight even in the absence of any aerodynamic lift.
\end{abstract}

DOI: 10.1103/PhysRevE.102.012403

\section{INTRODUCTION}

The phenomenon of aerial dispersal of spiders using strands of silk often called gossamer was identified and studied first with some precision by Martin Lister in the late 17th century [1], followed by Blackwall in 1827 [2], Darwin [3] on the Beagle voyage, and a variety of investigators since [4-7]. In modern parlance the behavior is known as ballooning. This term evokes a central question: does this mode of spider dispersal involve buoyancy forces, as the term suggests, or is it just a random process of aerial drift? Scientific investigation in the last several decades has largely dismissed the former possibility. Here we present evidence indicating that electrostatic buoyancy is a real and potentially important component of spider ballooning dispersal.

Development of the physics basis of spider ballooning to date has focused on the hypothesis that ballooning was an exclusively aerodynamic process, relying on lift generated through complex interaction between ballooning silk and the fluid dynamics of convective and wind-driven turbulence in the air. Humphrey [8] was the first to model ballooning as a fluid dynamic process, using a sphere (the spider) suspended by a rigid rod (the silk), achieving some success estimating observed characteristics of ballooning. Further refinements of the fluid dynamics approach have included flexible silk models $[9,10]$, and more sophisticated treatments of the effects of turbulence [11]. These models do yield lift in numerical simulations spider ballooning, but still appear to require sig-

\footnotetext{
*erica.morley@bristol.ac.uk
}

†gorham@hawaii.edu

Published by the American Physical Society under the terms of the Creative Commons Attribution 4.0 International license. Further distribution of this work must maintain attribution to the author(s) and the published article's title, journal citation, and DOI. nificant upward components to the local wind velocity distribution; whether actual wind momentum spectra provide the required distributions is still unproven, particularly for takeoff conditions. Even so, recent detailed observations of spider ballooning analyzed exclusively in terms of aerodynamic forces [12] provide plausible evidence that larger spiders can use multithread fans of relatively long silk, $3 \mathrm{~m}$ or more, to achieve takeoff and buoyancy in low winds within a certain turbulence regime. An example of multithread silk extrusion, shown in close proximity to the spinneret in a Linyphiid spider, is shown in Fig. 1.

Curiously, all of the aerodynamic models fail in one regard: they provide no mechanism to avoid entanglement of silk during the takeoff and float period. This issue becomes more acute for multithread silk fans, which are observed to splay out in a triangular pattern, even when potentially hundreds of threads are involved [13]. Multithread fans have been noted throughout the history of spider ballooning observations, and in all cases, observers are struck by the propensity of the silk fan to retain its shape and order [3,13], a fact that remains in tension with the putative turbulence required for buoyancy.

Under these conditions investigators have speculated that electrostatic charge on silk plays a role in avoiding entanglement, and producing the observed fanlike silk structures when multiple threads are emitted before launch [13]. Polymer filaments extruded from capillaries in textile and other filamentprocessing applications produce charges on the filament due to the phenomenon of flow electrification [14], thus some level of charge deposition on spider silk may be expected and may explain the silk's propensity to avoid entanglement. The molecular complexity of the spidroin dope compared to simple nonpolar hydrocarbons may also increase the possible range of electrification in spider silk, but as our results will show, if flow electrification is the source of the charge, it appears to be under rather tight control of the spider during the spinning stage. 


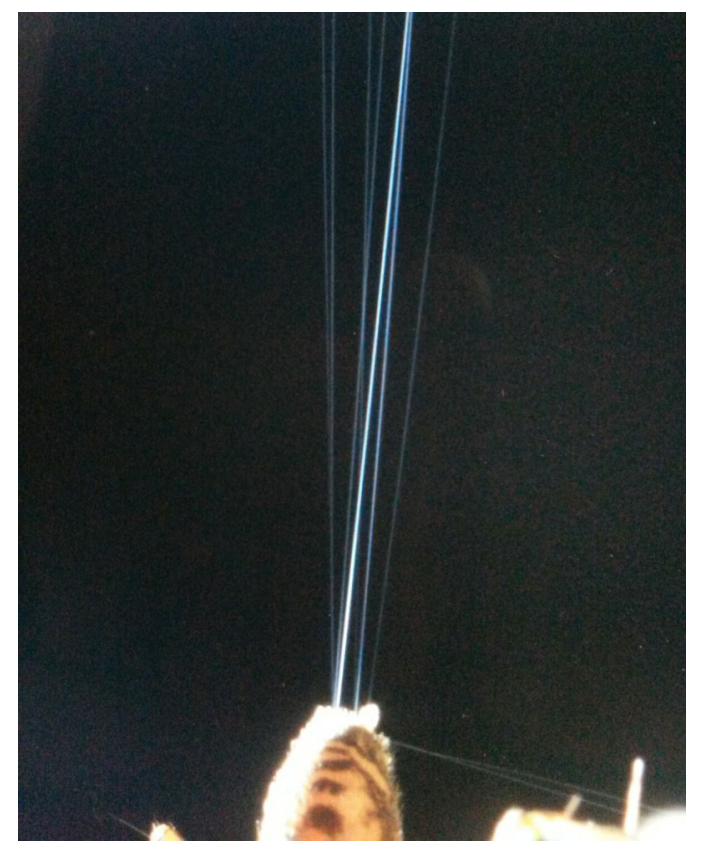

FIG. 1. Close-up image of a ballooning silk fan observed during activities related to the experiment reported here. (Photo: M. Hutchinson.)

Electrostatic forces are actively produced by Uloborus spiders when spinning fluffy cribellate silk [15]. This primitive type of silk forms puffs which have long been thought to hold their fluffy shape due to the repulsion of similarly charged threads, much like ballooning silk fans. There is thus evidence for spider control of the silk charge state during spinning, both in the development of silk fans among ballooners and in capture silk of Uluboris, but to date there are no direct measurements of the quantity of charge present in either of these cases.

The possibility of Coulomb force interactions in spider ballooning was considered with regard to the Earth's global electric field, along with considerations of the role of flow electrification in silk spinning, by Gorham [16]. However, no experimental investigation of the potential effect of the Earth's field in spider ballooning was undertaken until recently. In 2018, motivated by studies of the plausibility of physics underpinning such effects [16], and by the indications noted above that electrostatic fields may play some role in silk extrusion, Morley and Robert [17] found the first experimental confirmation in a laboratory setting that electric fields elicit ballooning behavior in spiders. These observations suggest that the Earth's electrostatic field [18-20], with a base value of typically $130 \mathrm{~V} / \mathrm{m}$, but with large variations in strength due to atmospheric activity, may play a role in producing lift utilized by spiders during ballooning. If so, spiders would be the first organisms known to make use of the Earth's electrostatic field for dispersal or any other behavioral activity.

We report here further investigation of the role of charged silk in spider aerial dispersal, using measurements of three carefully observed ballooning launches filmed within a laboratory chamber designed to be devoid of any air motion, containing a vertical electric field determined by internal parallel conducting plates at the top and bottom of the chamber. These launches were selected from a large range of ballooningrelated behaviors that were elicited by the presence of the vertical electric field, as shown in the controlled, blind study previously reported. [17]. Analysis of these data demonstrates that the only possible source of the acceleration observed in these events is the Coulomb force acting on charge contained on or entrained within the ballooning silk.

\section{EXPERIMENTAL METHODS}

\section{A. Morley and Robert experiment}

To provide context for the results reported here, we briefly review the previous results reported by Morley and Robert [17] and describe the test chamber.

Data acquired in 2018 by Morley and Robert use a clear $0.9 \mathrm{~m}^{3}$ plastic enclosure. The enclosure has a parallel plate configuration to establish a vertical electric field, with careful thermal and humidity control preventing air currents. A launch prominence was used, $25 \mathrm{~cm}$ high with a $2 \mathrm{~mm}$ diameter tip, made of non-conductive cardboard. Conductive $0.8 \mathrm{~m}$ square plates were positioned at the top and bottom of the chamber with $0.8 \mathrm{~m}$ vertical separation. A $37 \mathrm{~cm}$ diameter plastic dish filled with water around the base of the launch prominence prevented spiders from crawling away.

\section{Video and experiment protocol}

In this 2018 experiment, a group of 38 Erigone spiders, from a species known to be prolific ballooners, were tested for electric field response using the cardboard launch prominence. The spiders were subject to a series of tests with $0 \mathrm{~V}$ (control), $1000 \mathrm{~V}$, and $5000 \mathrm{~V}$ plate potentials. These plate potentials produce electric fields in the chamber, approximately $1250 \mathrm{~V} / \mathrm{m}$ and $6250 \mathrm{~V} / \mathrm{m}$, that are much larger than the Earth's fair-weather electric field, which typically ranges from 120 to $140 \mathrm{~V} / \mathrm{m}$. The higher values were chosen for the test as representative of disturbed weather fields in the locale from which the spiders originated.

During the tests, spider behavior was logged using video, and then scored blind according to two behaviors closely associated with ballooning: dragline drops from the prominence, followed by extrusion of ballooning silk, and tiptoeing, which is also followed by silk extrusion. Each spider received three trials, with voltage being switched on for a $2 \mathrm{~min}$ interval during the trial, after a 5 min initial acclimatization period. In each case, the launch site was carefully cleaned to remove any possible cues that might transfer between tests. The voltage sequences were randomized to avoid any patterns, and were unknown to the video observers. Each spider was also presented with only one treatment per day.

\section{Results}

The results of Morley and Robert showed a strong propensity for ballooning behavior with increasing electric field strength [17]. We summarize these results again in Fig. 2, where the test sequence has been ordered according to the voltage used (although in practice it was a random sequence). The correlation of ballooning-related behavior to the increasing plate voltage is clear and statistically compelling, with a final $p$ value of $p<10^{-6}$, close to $5 \sigma$ in Gaussian statistics. 


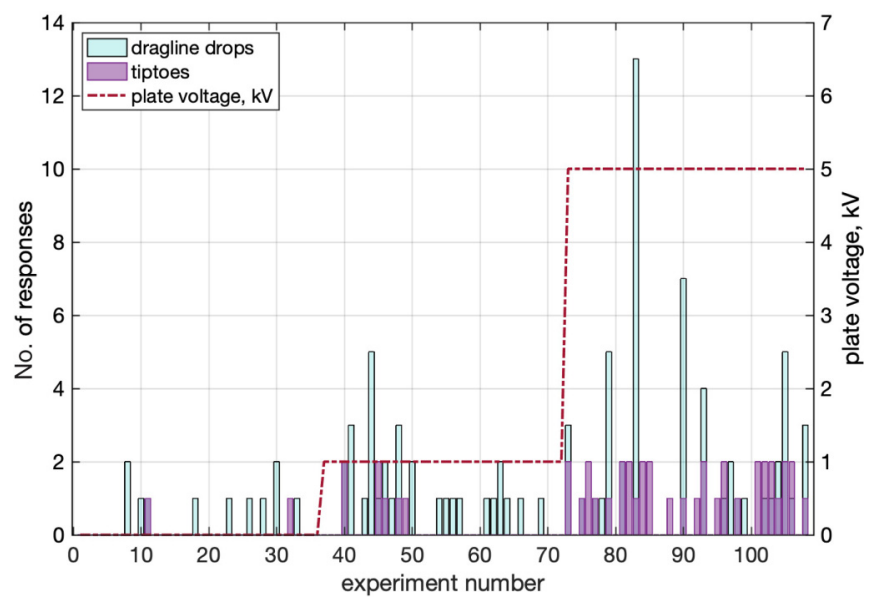

FIG. 2. Frequency of two well-documented preballooning behaviors as a function of external plate voltage in the spider test chamber.

This result from Morley and Robert [17] provides the first experimental demonstration that spiders initiate ballooning behavior in the presence of an electric field. This occurs in the absence of any significant air motion, which is precluded by the closed, temperature- and humidity-controlled chamber in which the tests were conducted.

Further tests using laser Doppler vibrometry (LDV) on the same spider species also established the location of the spiders' sensory organs: the trichobothria on their metatarsal legs [17]. These elongated hairlike features are known to be extremely sensitive to air currents [21], the data also showed that the trichobothria response to electric fields is distinguishable from that due to wind effects [17].

\section{Spider behavioral data not previously analyzed}

During these studies, a range of significant additional behavior relevant to ballooning was observed. Categorizing and quantifying these additional activities were not part of the original controlled experiment. To complement the work with the cardboard prominence, a new set of experiments using an aluminum-foil covered prominence were carried out, to provide a more extreme case of the field concentration around a conductive prominence. The new data is analyzed and presented in this paper. The increased electrostatic fields near the tip of the prominence led to an increase in ballooningrelated activities, which were also documented by video.

The observed behaviors included the following:

(1) Spiders extruding ballooning silk during high electric field periods, which became attached to the top or upper sides of the chamber, after which the spiders ascended the silk

(2) Dragline drops followed by extrusion of silk, followed by partial lift by the silk of the spider in the field, while still attached by the dragline

(3) Actual ballooning launches after tiptoeing and silk extrusion.

In all of these activities, it was qualitatively evident to observers at the time that electrostatic forces were clearly in play, and were associated with the ballooning silk, not simply electrostatic charge accumulated on the spiders themselves.
Activities of type (1) occurred several times, and although it was evident that electric fields were likely playing a role in extruding silk up toward the upper plate (as there was no other source of lift), there was no clear way to quantify the amount of lift that was produced.

Activities of type (2) were also quite common, and in principle, if the spider size and mass, and the shape of the catenary of the silk are known, it is possible to estimate the silk charge with reasonable precision. However, the ballooning silk was fine enough that, while the observers could clearly distinguish it, the video did not have adequate resolution to allow its shape to be quantified.

In one case, a spider that had extruded silk from a dragline drop then released the dragline and became free-floating in the chamber, and remained so for several high-voltage onand-off cycles [17]. The motion was captured by video, and a portion of it is used to quantify the charge in that instance here. Careful observation of this video also confirmed that the downward acceleration during the voltage-off condition was not consistent with rappelling, indicating the silk remained free-floating during this event.

Finally, two instances of activities of type (3), tiptoeing, followed by silk extrusion, followed by a clearly observed launch, were videotaped. In both cases the spiders rose rapidly out of the field of view of the video camera, which was then recentered, following the spiders' motions. The spiders' vertical rise abated in both cases within a short time due (evidently) to the impact of the silk with the upper plate, at which point the spiders would continue climbing, or might rappel down. To ensure that we measured only acceleration unaffected by either camera motion or the top plate, we used only those frames recorded before the camera began to move to estimate the initial acceleration of the spiders, and from this, to derive the required charge, given the electric field in the chamber.

Two frames from one of the launches showing a spider in the tiptoe position just at the moment of launch and several frames later is shown in Fig. 3. The spider body length is $2 \mathrm{~mm}$. The motion was not initially uniformly vertical as is evident in the frames, due to several-cm offsets in the silk axis relative to vertical. For this analysis we use only the vertical component, since motion in the horizontal direction is only measured in projection.

The group of four spiders involved in this subset of behaviors were measured for mass and body size, but unfortunately the data on individual spiders were lost, and only the value of $0.9 \mathrm{mg}$ for the average mass of the four spiders was preserved. Based on the video scales, we conclude that the variance of the group is relatively small, but it does constitute an additional uncertainty, which we include by variational analysis below.

\section{ANALYSIS FRAMEWORK}

In a uniform vertical electric field $\mathbf{E}=E_{0} \hat{z}$, a spider of mass $m$, cross-sectional area $A$, charge $q_{b}$ on the spider body, and total charge $q_{s}$ on the extruded silk, will experience the Coulomb force $\mathbf{F}_{C}=\left(q_{b}+q_{s}\right) \mathbf{E}$, and a gravitational force $\mathbf{F}_{g}=-m g \hat{z}$, with $g=9.8 \mathrm{~m} \mathrm{~s}^{-2}$. Once the motion begins, a drag force $\mathbf{F}_{d}$ will also develop. 

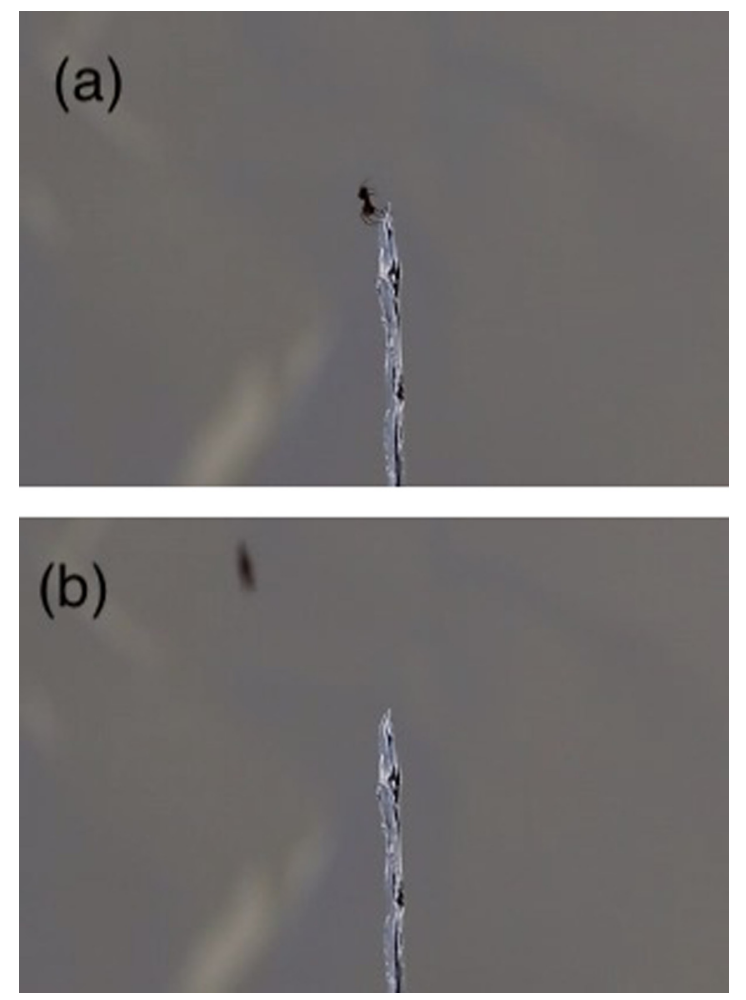

FIG. 3. Two examples of frames of the videos used for the acceleration measurements. (a) Tiptoeing spider just at the moment of launch, with fine ballooning silk already deployed and extended vertically, as seen by observer although unresolved in the video. (b) Spider in motion, just before leaving the frame $160 \mathrm{~ms}$ later.

The form of the drag force depends on whether the velocity of the spider is in the viscous drag regime, or in the pressure drag regime. In the viscous drag regime, we use Stokes drag as a model,

$$
\mathbf{F}_{d, S}=6 \pi \eta r \mathbf{v}
$$

and for pressure drag,

$$
\mathbf{F}_{d, P}=-\frac{1}{2} \rho C_{d} A v^{2} \hat{v} .
$$

Here $\eta=1.85 \times 10^{-5} \mathrm{~kg}(\mathrm{~m} \mathrm{~s})^{-1}$ is the dynamic viscosity of air at standard temperature and pressure (STP), $r$ is the equivalent spherical radius of the spider, $\rho=1.2 \mathrm{~kg} \mathrm{~m}^{-3}$ is the sea level density of air at $\mathrm{STP}, \mathbf{v}, v, \hat{v}$ are the velocity, speed, and unit velocity vector of the spider, $C_{d}$ is the drag coefficient, and $A$ the cross-sectional area.

Which of these terms dominate will depend on the Reynolds number of the flow around the spider

$$
R_{e}=\frac{\rho v d}{\eta}
$$

where $d$ is a characteristic dimension, $d \simeq \sqrt{A}$. For the spiders considered here,

$$
R_{e}=6.49\left(\frac{d}{1 \mathrm{~mm}}\right)\left(\frac{v}{10 \mathrm{~cm} / \mathrm{s}}\right),
$$

and it is evident that except for the earliest part of the motion, $R_{e}>1$, pressure drag will prevail. Since Stokes drag may still play a role during the initial launch, we do not neglect it in the model. Spiders are not streamlined in their cross section, so we expect the drag coefficient to be $C_{d} \gtrsim 1$; in fact we will use experimental data on spider free fall to estimate reasonable values of $C_{d}$ in the analysis below.

From Newton's second law, the sum of the forces is then

$$
\sum_{i} \mathbf{F}_{i}=m \mathbf{a}=\mathbf{F}_{C}+\mathbf{F}_{g}+\mathbf{F}_{d},
$$

where $\mathbf{a}$ is the resulting acceleration. This equation is essentially the same as the result in Ref. [8] with the additional Coulomb and Stokes drag forces included, wind velocity $\mathbf{v}=0$, and neglecting the $O\left(10^{-3}\right)$ correction for the air displacement of the spider. Inserting the forms for each of the terms and assuming all forces are acting only vertically as determined by the test chamber

$$
m a=Q E_{0}-m g-\frac{1}{2} \rho C_{d} A v^{2},
$$

where total charge $Q=q_{b}+q_{s}$. We can solve this equation for $Q$ if the the acceleration is a measured quantity:

$$
Q=E_{0}^{-1}\left[m(a+g)+\frac{1}{2} \rho C_{d} A v^{2}\right] .
$$

The first term in brackets above, $m(a+g)$ is dominated by the gravitational acceleration for any $a<1 \mathrm{~m} \mathrm{~s}^{-2}$. For spiders of mass $m=1 \mathrm{mg}$ this term will have a magnitude $F \sim 10 \mu \mathrm{N}$. For any spider with $d<10 \mathrm{~mm}$, the drag force term will be no more than $10 \%$ of the gravitational term for any velocity below about $40 \mathrm{~cm} / \mathrm{s}$, well within the apparent range of velocities observed. So to first order, we can neglect the drag forces, and the resulting charge equation becomes

$$
|Q|=[m(a+g)] /\left|E_{0}\right| .
$$

Again since the observed accelerations $a<<g$, the resulting charge depends to first order only on the spider mass, and for $|E| \simeq 7.5 \mathrm{kV} / \mathrm{m}$ in the plateau region we find a lower limit on the total charge of

$$
|Q| \geqslant \frac{m g}{\left|E_{0}\right|}=1.3 \mathrm{nC}\left(\frac{7.5 \mathrm{kV} / \mathrm{m}}{E_{0}}\right)\left(\frac{m}{1 \mathrm{mg}}\right) .
$$

The lower limit arises since the other terms initially neglected, drag, nonvertical motion, and the actual observed (nonzero) acceleration of the spiders, will lead to a larger charge.

The fraction of $Q$ that resides on the silk versus the body of the spider is not yet determined. However, measurements of induced charge on houseflies [22] and bees [23,24] provide a scale for the electrical capacitance of insect bodies, with total absolute charge from a wide variety of activities ranging from 40 to $600 \mathrm{pC}$. Assuming spider carapace properties are electrically similar, the charge capacity will be determined by the total area, For honey bees, this is estimated at $3.3 \mathrm{~cm}^{2}$ on average [25]. By comparison, the spiders used in this experiment are much smaller, with an estimated area of order $6 \times 10^{-2} \mathrm{~cm}^{2}$. We can expect the range of possible spider body charge to be $0.7 \mathrm{pC} \leqslant q_{b} \leqslant 10 \mathrm{pC}$, and thus the spider body charge will likely contribute less than $1 \%$ of the total Coulomb force for spiders that attain lift. We do not neglect the body charge in what follows; instead we will include both $q_{s}$ and $q_{b}$ into the final fitted results below.

Equation (1) sets the scale of the of the silk charge, but under idealized conditions. In practice, the silk charge may be 

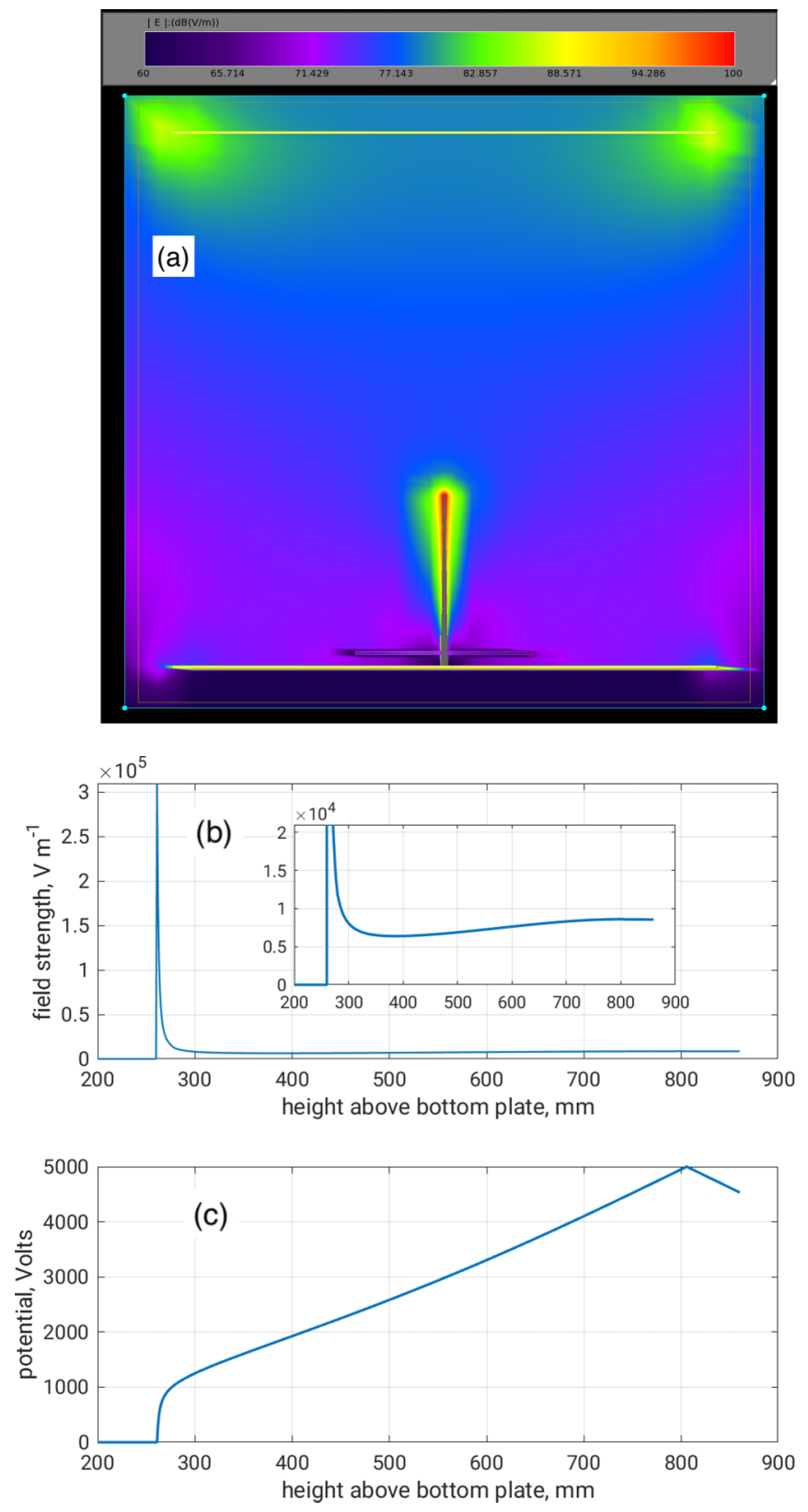

FIG. 4. (a) Electric field estimated by Remcom XFDTD model. (b) Vertical section through the modeled field above the tip of the conductive launch point. (c) The integrated potential through the same section.

distributed and the field is nonuniform. To estimate the threedimensional electric field configuration, we used a commercial finite-difference-time-domain electrostatic solver, Remcom XFDTD, version 7.7 [26], and the results are shown in Fig. 4. The system was modeled with precision in all significant details, except for the actual surface structure of the aluminum foil prominence, which had a random texture due to the fabrication of the prominence by hand, as evident in Fig. 3. Thus we expect that the model will accurately reproduce the fields on mid- and large scales within the chamber, but precise details in close proximity to the foil surface will have increased uncertainty.
Under these electric field conditions, estimating the force requires an integral over the charge distribution and the nonuniform field. The net vertical force for a nonuniform field $E(z)$ and charge distribution $q(z)$ is given by

$$
m a=\int_{z}^{z+L} q(z) E(z) d z-m g-F_{d},
$$

where $F_{d}$ is the relevant drag force (which may be a combination of Stokes and pressure drag). This equation can be written in the canonical form for coupled ordinary differential equations [27]:

$$
\begin{aligned}
\frac{d v}{d t} & =\frac{1}{m} \int_{z(t)}^{z(t)+L} q(z) E(z) d z-m g-F_{d}, \\
\frac{d z}{d t} & =v(t),
\end{aligned}
$$

where the second equation defines the coupling between vertical position and velocity. The charge distribution function $q(z)$ can be approximated as a $\delta$ function at the spider location for the body charge, plus a distribution for the silk charge, thus $q(z)=q_{b} \delta\left(z-z_{b}\right)+q_{s}(z)$.

Equations (3) are not straightforward to solve analytically, and given that the charge distribution $q_{s}(z)$ is unknown and the field distribution has no analytical model, it must solved numerically for assumed charge distributions. We consider two cases for $q_{s}(z)$ : first, a uniform charge distribution per unit length of silk, $q_{s}(z)=\left(Q-q_{b}\right) / L$, where $Q$ is the total charge and $L$ the length of the silk. This distribution may be expected for charge that is entrained or deposited along the silk, under conditions where the initial electrical conductivity of the silk is low enough that charge mobility can be neglected on the timescale of the launch.

At the other end of the scale is charge mobility that is high enough to allow charge to flow continuously toward the distal end of the silk in response to the external electric field, given the intrinsic capacitance and conductance of the silk as it is freshly extruded from the spinneret. For this second case we assume a point charge $q_{s}(z)=\left(Q-q_{b}\right) \delta\left(z-z_{s}\right)$ located at the centroid of some finite segment of charge near the upper end of the silk, where the field is nearly constant.

We then evolve the equations of motion using a fourthorder Runge-Kutta (RK4) method to iteratively fit the position and velocity for the three acceleration events we have observed.

\section{RESULTS}

\section{A. Video frame calibration}

Video frame scales were calibrated by using the average spider body length derived from the average $0.9 \mathrm{mg}$ mass, estimated from statistical studies of spider mass versus body length for a large number of spiders of similar type [28]. The implied mean body length for the spiders involved in the launches is $\sim 2.0 \mathrm{~mm}$, consistent with the measured range of $1.8-2.8 \mathrm{~mm}$ of the full group of 38 spiders. We address the effects of this uncertainty later in this section.

Positions and their errors determined from centroids of the spider body position for each frame. The frame rate is 25 frames per second, giving $40 \mathrm{~ms}$ per frame. For the force 

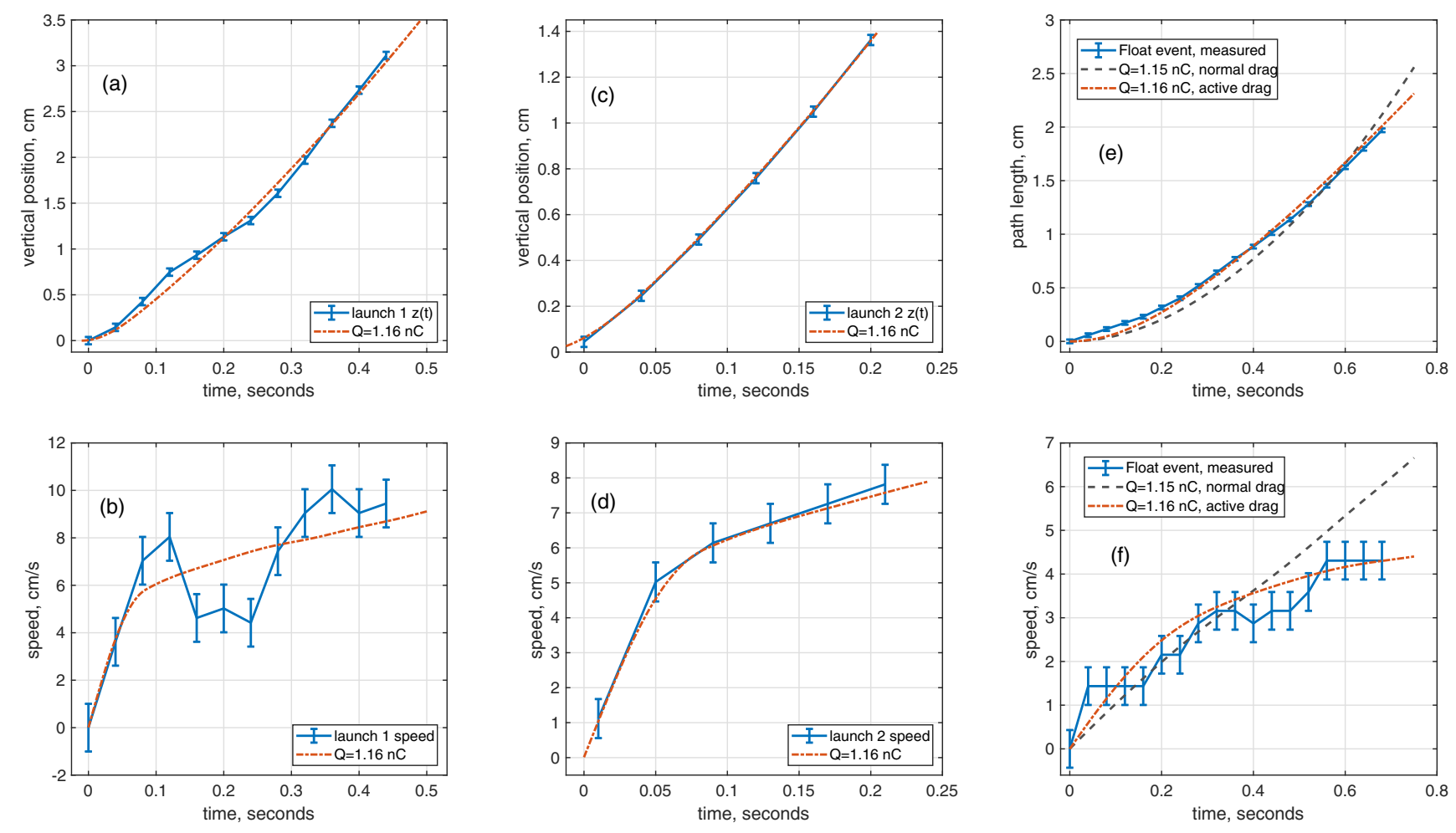

FIG. 5. (a) Modeled and measured position for the first observed launch, denoted MVI 1407. (b) Modeled and measured speed for MVI 1407 launch. (c) Modeled and measured positions for the second observed launch, denoted MVI 1503. (d) Modeled and measured speeds for MVI 1503. (e) Modeled and measured positions for the float event. (f) Modeled and measured speeds for the float event.

equation integrated using the RK4 method, we included gravity, the Coulomb force, and both viscous and pressure drag. An additional term accounting for stretch in the ballooning silk under tension just prior to launch was estimated using a Hooke's law spring force, $F=k \Delta z$, with spring constant $k$ dropping to zero once the initial strain was relaxed. The total range of motion that could be observed without losing calibration due to camera motion was $1.5-3.5 \mathrm{~cm}$. While a larger range of motion would certainly be preferred, the standard errors on positions measurements were fractions of a millimeter, so this limited range of motion still provides clear constraints on the forces involved.

\section{B. Exclusion of uniform charge density}

In our results, continuous uniform charge distributions produced solutions in which the spider oscillated around the launch point with a period of about $0.5 \mathrm{~s}$. This is due to the fact that the strong local fields at the launch point, coupled with the charged silk in very close proximity, initially dominate the acceleration, forcing the fitted linear charge density to a relatively low value in order not to exceed the observed speeds. Then, when the spider leaves the immediate vicinity of the launch point, within less than $1 \mathrm{~cm}$, the precipitous drop in the field causes the lift force due to the underestimated charge density to fall below that of gravity. The spider then falls back down until the strong field region is entered. This behavior is completely inconsistent with the observed acceleration, and we thus exclude a uniform distribution of charge as a viable solution.
In fact, as noted above, we believe that fresh ballooning silk allows for charge mobility high enough to ensure that the charge migrates rapidly away from the strong local electric field at the launch point. A recent study of the electrical conductivity of spider silk found it to be a very strong function of local relative humidity, increasing by more than three orders of magnitude, from $<10^{-6}$ to $\sim 10^{-3} \mathrm{~S} / \mathrm{m}$ over the range of $30 \%$ to $70 \%$ relative humidity, a remarkable change [29]. These measurements were made with dragline silk tested well after harvesting; to our knowledge no measurement of the electrical conductivity of fresh ballooning silk has been published.

We hypothesize that freshly extruded silk, which has only just been dehydrated within moments of leaving the spinneret, may have a relatively high conductivity during its initial phase. Under the high launch point field concentration, charge would naturally be driven away from the launch point and toward the end of the silk, which would be subject to far more uniform fields in the upper region of the chamber. Our results are in fact consistent with either a concentrated charged region near the silk upper end, or a distributed silk charge within the plateau region of the field, as seen in Fig. 4.

\section{Model fit results}

The results of these RK4 model fits, along with the measured data, are shown in Fig. 5, for both vertical position relative to the start point (upper frames), and the vertical speed (lower frames). The nonlinear nature of the motion is evident in each case, both in position and speed. The fitted charge, 
with negligible statistical errors, is $q_{s}=1.15-1.16 \mathrm{nC}$, consistent with Eq. (1) for the average $0.9 \mathrm{mg}$ mass of the spiders involved. The fitted value for the spider body charge is also consistent for all three data sets: $q_{b}=3 \pm 0.5 \mathrm{pC}$, which is of the same order of magnitude as the linear charge density, about $10 \mathrm{pC} / \mathrm{mm}$, implied by the electric field models at the upper end of the aluminum foil prominence.

These were values determined using a grid-search minimization of the $\chi^{2}$ function for each of the three ballooning events:

$$
\chi^{2}=\sum_{i=1}^{N} \frac{\left[z_{o}\left(t_{i}\right)-z_{m}\left(t_{i}\right)\right]^{2}}{\sigma_{z}^{2}},
$$

where $N$ is the number of frames, $z_{o}\left(t_{i}\right)$ is the observed vertical position at time $t_{i}, z_{m}\left(t_{i}\right)$ is the modeled position at time $t_{i}$, and $\sigma_{z}, \sigma_{u}$ are the estimated standard errors in position and speed, respectively. The model positions are given by the numerical solutions to Eq. (3).

Statistical uncertainties are typically $\sigma_{z}=0.4$ to $0.5 \mathrm{~mm}$ in the video frame position estimates, which translates to about $\sigma_{u}=1 \mathrm{~cm} / \mathrm{s}$ uncertainty in the speeds. Since the speeds are derived from the positions, they do not provide independent information for the $\chi^{2}$ minimization but are shown in Fig. 5 because they show more clearly the transient effects: the local repulsion of the spider from the launch point due to the body charge, and the short duration of the snap-back of the stretched thread, both of which contribute to the early higher acceleration of the spider at launch. These effects are absent in the float event, since the spider was already at least $6 \mathrm{~cm}$ away from the launch prominence when the acceleration started, well outside of the highly enhanced field at the tip of the prominence.

In these events the spiders typically rose an additional distance of $20 \mathrm{~cm}$ or more beyond the vertical range we analyze here, but the requirements for calibration of the scale of the motion lead us to restrict the data to the initial period before camera motion began. Despite this restriction, we emphasize here that this motion is completely inconsistent with any other force available to the spiders. There was no thermal gradient to produce any significant air motion within the chamber, certainly not at the speeds of $4-8 \mathrm{~cm} / \mathrm{s}$ as observed. Spiders were watched carefully to ensure that no silk had attached to the chamber walls or top, and the motion observed in these launches involved no climbing actions by the spider. The only known source of lift in this case is the Coulomb force, and the observed motion requires that this force must be primarily exerted on the upper portion of the silk, where we conclude the charge resides.

\section{Systematic uncertainties}

Systematic uncertainties in these results include (a) the unknown drag coefficients of the spiders, (b) possible pendulum motions immediately after launch due to off-axis electric field effects near the launch point, (c) uncertainties in silk strand length, and (d) the uncertainty in the video size scale.

For (a) we used values consistent with spider free-fall measurements made by Suter [30], which imply surprisingly large drag coefficients, $C_{d} \gtrsim 3$, based on estimated cross-sectional body areas, perhaps because of the complex shape, including the legs, for which the effective area is difficult to estimate. We varied the pressure drag coefficient $C_{d}$ by factors of two around our adopted baseline value $C_{d}=3$ with no effect on the results. Other systematics were also checked by variational methods, and the resulting charge estimates were found to be quite robust to these variations.

As noted above, pendulum-like motions were observed in the early part of one of the launches; we compensate for (b) by using only the vertical component, which is conservative in that it underestimates the total acceleration. To address (c), an approximate estimate of strand length $L \simeq 0.4$ to $0.5 \mathrm{~m}$ was obtained by observing at what elevation the spider motion was abated, but we also did not assume any strand-length dependent parameters, solving for total charge under the assumption only that there was charge migration to its upper half, in the region where the electric field plateaued, during extrusion. As we have also noted above, the observed motion is not consistent with a uniform charge distribution.

The effect of the scale uncertainty [(d)], which we expected to translate linearly to an uncertainty in the resulting charge, was in fact quite small, as we found by fitting for the charge while varying the scale within the range of the uncertainty. We attribute this to the fact that the upward spider vertical accelerations we observed, while clearly evident, were still small in magnitude compared to the gravitational acceleration, and thus errors in these only affect the resulting charge at second order, since to first order [e.g., Eq. (1)] the charge is independent of spider acceleration for $a \ll g$.

\section{E. Quality of the model fits}

For the two launches, the RK4 numerical model fits the overall motion well. The speed in the 1407 launch includes some variation, probably due to some pendulum motion of the spider, that is not yet very well modeled, due to lack of precise knowledge of the silk shape, but once the pendulum motion abates, the model again aligns with the data.

For the float event, the observed motion appears to include a deceleration in excess of the expected spider drag, and indicates some deficiency in the model, although the fitted charge remains quite similar. We thus also show the results of an Ansatz model in which the spider pays out silk during the upward motion. We model this as an additional draglike term, which we denote as "active drag." In practice its magnitude is equivalent to an increase of an order of magnitude in the pressure drag coefficient but results in a negligible change in the magnitude of the silk charge required by the fit. This is illustrative but not conclusive-it remains one of the open questions of the resulting motion. Despite these moderate discrepancies the measured motion shows clear and compelling evidence that nanocoulomb charges must reside on ballooning silk.

In Table I we show a summary of the fitted charges for the three ballooning events, with two drag scenarios considered for the final float event. The table includes estimates for the combined systematic + statistical errors, along with the final $\chi^{2}$ and the number of degrees of freedom in each case. In the float event the discrepancies in the fits are evident in the final $\chi^{2}$ values; although the active drag is a much better fit to the data, the model is still not fully commensurate with the data. 
TABLE I. Results of charge fitting for the three ballooning events reported here.

\begin{tabular}{|c|c|c|c|c|}
\hline $\begin{array}{l}\text { Ballooning } \\
\text { event }^{\mathrm{a}}\end{array}$ & $\begin{array}{l}\text { Estimated } \\
\text { charge, } \mathrm{nC}\end{array}$ & $\begin{array}{c}\text { Total } \\
\text { error, } \mathrm{nC}^{\mathrm{b}}\end{array}$ & $\chi_{\min }^{2}$ & $\mathrm{DOF}^{\mathrm{c}}$ \\
\hline MVI1407 & 1.155 & 0.02 & 49.9 & 11 \\
\hline MVI1503 & 1.158 & 0.02 & 0.95 & 5 \\
\hline Float, normal drag ${ }^{\mathrm{d}}$ & 1.154 & 0.02 & 544 & 17 \\
\hline Float, active drag ${ }^{\mathrm{e}}$ & 1.160 & 0.02 & 76.7 & 17 \\
\hline
\end{tabular}

a Direct launches are indicated by the video recording number; the "Float" case involved a free-floating spider.

${ }^{\mathrm{b}}$ Combination of estimated statistical and systematic errors.

${ }^{\mathrm{c}}$ Degrees of freedom for the position $\chi^{2}$ as given in Eq. (4).

${ }^{\mathrm{d}}$ Best estimate for simple Stokes + Pressure drag.

${ }^{\mathrm{e}}$ Assumes spider actively extrudes silk after launching to reduce acceleration.

For MVI1503, the fit is reasonably good, and for MVI1407, the tension in the fit comes mainly from fluctuations in the trajectory, likely due to pendulum motion.

These results represent direct measurement of such charges via acceleration in a controlled environment. It is surprising that the values measured all fall within a very small range. This raises important questions about the silk extrusion: do ballooners have the ability to tune or even modify in real time amount of charge on their ballooning silk, depending on the environment? If so, how is the charge manipulated? Is it embedded onto the silk by the extrusion process itself, or entrained from charge at the launch point?

\section{F. Space charge issues}

The quantity of charge observed corresponds to about $7 \times 10^{9}$ excess electrons. This implies that spider silk is a form of electret, a material able to store and retain free charge for some period long compared to the initial charging time. The observed diameter of the multistranded ballooning silk in this experiment was typically $\sim 340 \mathrm{~nm}$, as confirmed by scanning electron microscopy, examples of which are shown in Fig. 6. If we assume the excess electrons are concentrated uniformly in the upper $40 \%$ of the silk length, in a segment with $\ell=20$ $\mathrm{cm}$, the resulting space charge density at the nanoscale is $\rho=1.1 \times 10^{-3}$ electron $\mathrm{nm}^{-3}$. We can then estimate the excess charge per spidroin molecule. Following Erickson [31], the folded protein volume $V$ can be approximated as

$$
V\left(\mathrm{~nm}^{3}\right) \simeq 1.212 \times 10^{-3}\left(\mathrm{~nm}^{3} \mathrm{Da}^{-1}\right) \times M(\mathrm{Da})
$$
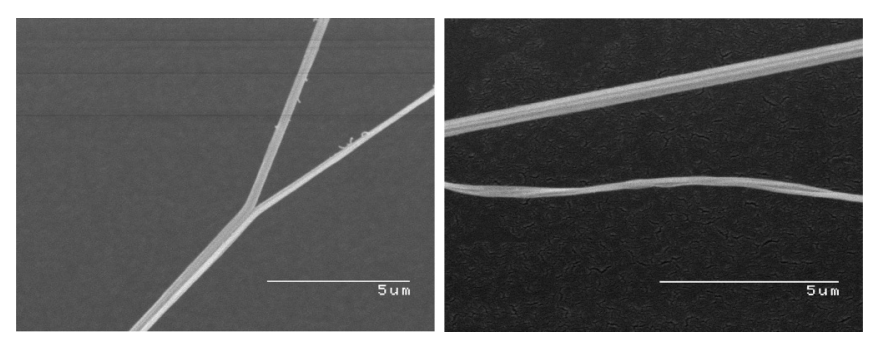

FIG. 6. Two SEM images from ballooning silk gathered during the experiment activities reported here. (Credit: E. Morley.) for molecular mass $M$ in daltons. For a mean molecular mass of $\sim 300 \mathrm{kDa}$ for spidroin, the resulting volume is $364 \mathrm{~nm}^{3}$, implying 0.42 excess electrons per folded spidroin molecule. This is a quite low charge excess for these large molecules, presenting no obvious problems given the typical $\sim 3500$ amino acid constituents of silk spidroin.

However, the implied charge density of the silk, viewed as an electret in this case, assuming an effective radius for the ballooning silk of $r_{e} \simeq 170 \mathrm{~nm}$, is $\rho=64 \mathrm{kC} \mathrm{m}^{-3}$. This is much larger than the bulk charge density of typical polymer electrets, which can reach $2.5 \mathrm{kC} \mathrm{m}^{-3}$ [32]. In fact, if the silk is conductive enough to allow charge migration during the initial spinning of the ballooning thread, a period of order a few seconds at most, it may be assumed that the charge would migrate to the surface, and thus yield near-surface charge densities in even stronger tension with those seen in polymer electrets.

There are several ways in which this tension may be mitigated. First, recent work has demonstrated space charge densities as high as $100 \mathrm{kC} \mathrm{m}^{-3}$ in micro-electrets in the form of $500 \mathrm{~nm}$ diameter silica-based spherules [33]. As the authors of this study demonstrate, such charge densities are much easier to accommodate in micrometric scale bodies with low dimensionality, a feature that spider silk shares with this example.

Second, the authors of this study on micro-electrets also note that the structure of the spherules used, which involves a process that builds up the spherule from smaller silica nanoparticles, yields a higher internal surface area for charge accumulation than a homogeneous material. The structure of spider silk, spun from series of $\sim 100$ nanometer-scale fibrils (as seen in Fig. 6), also yields a significantly larger surface area than the equivalent single-strand cylinder. The corresponding charge-trapping efficiency of silk, with its complex amino-acid structure, is thus likely to be much higher than a normal bulk polymer electret.

Finally, while the conductivity required of the fresh silk in order for space charge to propagate longitudinally to the distal end of the strand (as required by our observations) is much higher than for normal dielectrics, it is still far below that of metals. The conductivity range for silk as noted above, from $<10^{-6}$ to $\sim 10^{-3} \mathrm{~S} / \mathrm{m}$ over the range of $30 \%$ to $70 \% \mathrm{RH}$ [29], falls in the transition region appropriate for semiconductors; thus for example, the conductivity of amorphous silicon can be as low as $10^{-3} \mathrm{~S} / \mathrm{m}$, and that of cadmium sulfide as low as $10^{-5} \mathrm{~S} / \mathrm{m}$. Space charge effects in such materials are common and in fact help to define the nature and behavior of such materials [34].

It is worth also noting that the spinning process itself, in which the final stage of motion of the spidroin dope through the spinneret involves dehydration of the dope via ionic effects at the walls of the spinneret tubule, may yield a material with a much higher conductivity close to the central axis of the fibroin than at its perimeter. As noted in the introduction, flow electrification is one mechanism for creating the charge, but in the spinning of simple polymeric fibers, there is no equivalent dehydration phase. Flow electrification with a uniform conductivity typically produces a radial profile with space charge concentrated near the walls of the flow tube [35,36]; in the case of spinneret flow, a conductivity gradient could significantly modify the result. 
Such an asymmetric conductivity profile could in fact yield enhanced longitudinal migration of the space charge, while limiting surface charge density due to the decreased mobility near the perimeter. Under these conditions, where the hydration state of the silk is closely coupled to its conductivity, the relative permittivity $\epsilon_{r}$ is also likely to have a strong radial dependence, since $\epsilon_{r} \sim 80$ for water. Such a permittivity gradient would also tend to reduce the radial space charge mobility due to the polarization of the silk, which would reduce the apparent radial electric field.

In summary, then, while the space charge density implied by our observations are quite high, further studies of the nanoscale structure, permittivity, and conductivity of freshly spun silk are necessary to understand the physical nature, nanoscale and macroscale distribution, and mobility of the charge.

\section{IMPLICATIONS FOR FAIR-WEATHER FIELD CONDITIONS}

The electric field conditions used in these experiments was based on conditions observed in active or disturbed weather, with ambient fields that are much stronger than the Earth's fair-weather APG of $\sim 130 \mathrm{~V} \mathrm{~m}^{-1}$. One of the important questions this raises is whether spiders make use of the fairweather field at all, or do they only make use of silk charge under high APG conditions.

We have modeled fair-weather APG conditions with a plant stem prominence that has parameters similar to plant stems in nature, with electrical properties determined largely by the water-based electrolytes in their sap. Whole-stem conductivities for plants fall in a typical range of $\sigma=$ $10-100 \mathrm{mS} / \mathrm{m}$, with relative dielectric constants of order $\epsilon_{r}=$ $10-50$, again strongly affected by water content $[37,38]$. We estimate the near-surface conductivity, which matters for the surface electric field development, to approach that of saline water: $\sim 4 \mathrm{~S} / \mathrm{m}$; we use $\sigma=1 \mathrm{~S} / \mathrm{m}$ as the surface conductivity, and $\epsilon_{r}=50$, accounting for surface adsorption of water vapor in a humid environment. The same plate geometry as the simulation above is used, except that we use a top plate voltage of $110 \mathrm{~V}$, giving an ambient field of about $140 \mathrm{~V} / \mathrm{m}$, although with $10 \%$ nonuniformity due to the finite plate size. The plant stem is assumed to be a $30 \mathrm{~cm}$ high conical frustum, $0.4 \mathrm{~cm}$ in diameter at the bottom, and $1 \mathrm{~mm}$ in diameter at the top.

Figure 7 shows the results of this simulation, with profiles of the resulting electric fields around the tip of the plant stem, both in the transverse and vertical directions. The small diameter of the dielectric tip strongly magnifies the field, although much less so than a conducting tip would. The resulting fields approach $1 \mathrm{kV} / \mathrm{m}$ in a region several $\mathrm{mm}$ around the tip, with some irregularities due to the mesh structure. This field appears to be well within the sensor range of the spiders tested previously by LDV methods when on the cardboard prominence, where fields as low as $400 \mathrm{~V} / \mathrm{m}$ showed a clear response well above background noise [17]. Thus it appears that at least from a sensory perspective, these spiders are sensitive to changes at the field levels of Earth's fair-weather APG.

The nanocoulomb charges observed in these experiments would produce very small lift, $\sim 2 \%$ of their weight, for a
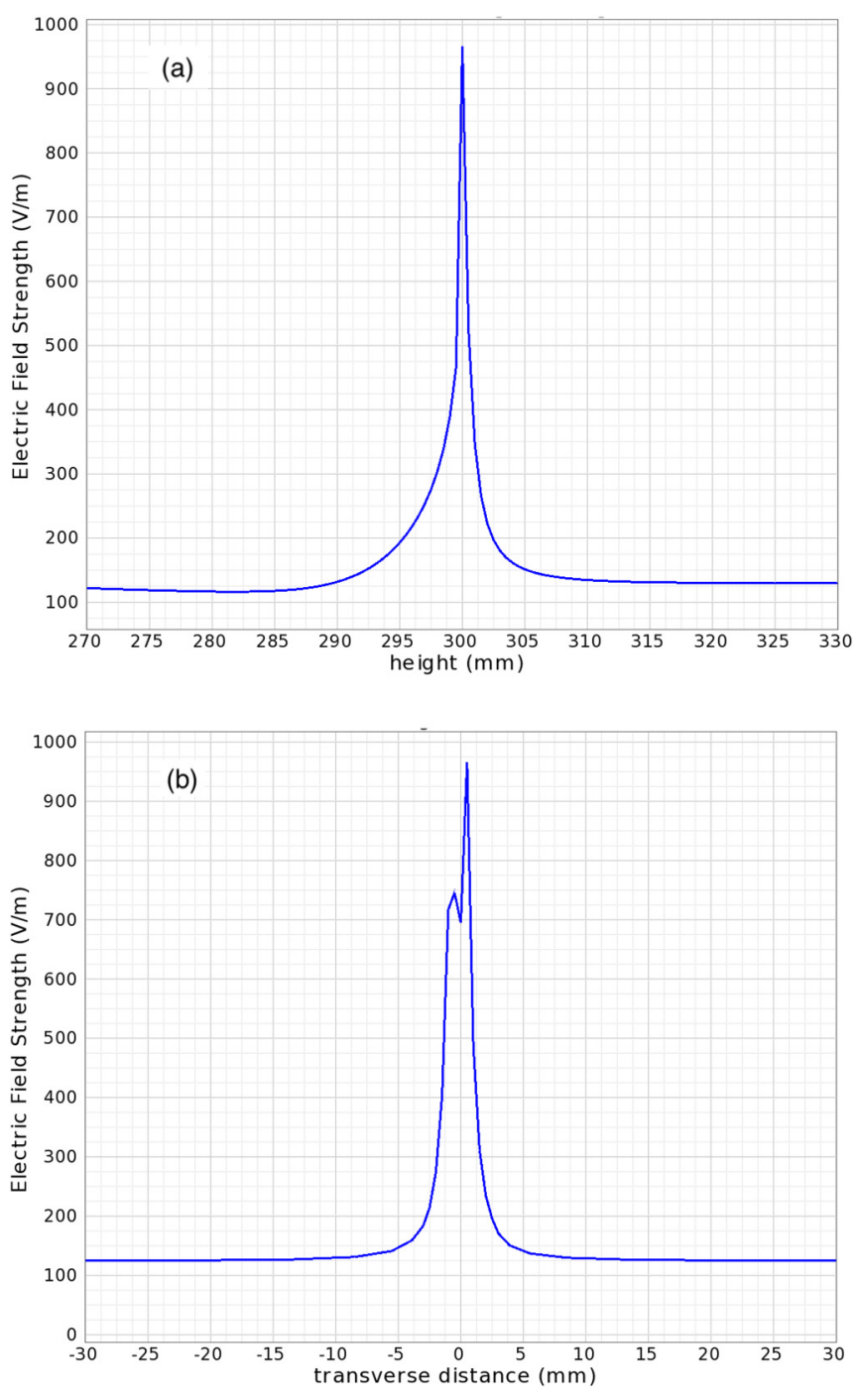

FIG. 7. (a) Vertical profile of Remcom XFDTD simulation of fair-weather APG fields along a simulated plant stem. (b) Horizontal profile through the tip of the same stem.

spider with a single ballooning silk strand in the fair-weather field, however. Thus a crucial question arising from these results is whether spiders modify their behavior and silk extrusion process to adapt to lower fields. Certainly one approach is to use longer silk, for example ballooning silks up to $3 \mathrm{~m}$ long have been observed in natural ballooning of larger spiders [12]. Assuming a linear increase in total charge, an order of magnitude more lift may be possible by this method alone for the spiders considered here. If in turn another half-dozen or more such silks were extruded, as implied by Fig. 1, the Erigone spiders observed in our experiment would achieve positive buoyancy even in the fair-weather atmospheric potential gradient, without the aid of any wind.

In conclusion, we have measured the charge state of spider ballooning silk by observing the acceleration it produced on the spider+silk system in an electric field. These accelerations were observed in controlled conditions in which no wind was present, thus the lift produced, and the resulting launches of the spiders, was due purely to electrostatic forces on the silk 
itself, since the spider body charge could not produce the motion seen.

These observations, combined with previous measurements showing that the presence of electrostatic fields elicits ballooning responses, and that spiders have sensory organs to detect both static and changing electrostatic fields [17], gives credence to the proposal that spider ballooning is not a purely aerodynamic process, but involves an electrostatic component. These results also strongly suggest that spiders have developed adaptations to directly exploit the Earth's atmospheric potential gradient in ballooning dispersal behavior; if confirmed they would become the only known organisms of any kind to make active use of the global electric field. Further work is still needed to determine the interplay between aerodynamic and electrostatic forces, and their relative contributions under different circumstances.

\section{ACKNOWLEDGMENTS}

The experimental work was funded by a research grant to E.L.M. by the Association for the Study of Animal Behavior. E.L.M. would like to thank Penelope Fialas for her assistance in data collection and Daniel Robert for providing lab space and equipment.
[1] A. M. Roos, Web of Nature: Martin Lister (1639-1712), the First Arachnologist (Brill, Leiden, 2011).

[2] J. Blackwall, Observations and experiments, made with a view to ascertain the means by which the spiders that produce gossamer effect their aërial excursions, Trans. Linnean Soc. London 15, 449 (1827).

[3] C. Darwin, A Naturalist's Voyage: Journal of Researches into the Natural History and Geology of the Countries Visited during the Voyage of H.M.S. Beagle round the World under the Command of Captain Fitz Roy, R.N., 2nd ed. (John Murray, London, 1889).

[4] E. Duffey, Aerial dispersal in a known spider population, J. Anim. Ecol. 25, 85 (1956).

[5] J. R. Bell, D. A. Bohan, E. M. Shaw, and G. S. Weyman, Ballooning dispersal using silk: World fauna, phylogenies, genetics and models, Bull. Entomol. Res. 95, 69 (2005).

[6] G. A. Weyman, K. D. Sunderland, and P. C. Jepson, review of the evolution and mechanisms of ballooning by spiders inhabiting arable farmland, Ethol. Ecol. Evol. 14, 307 (2002).

[7] A. Reynolds, Beating the odds in the aerial lottery: Passive dispersers select conditions at takeoff that maximize their expected fitness on landing, Am. Nat. 181, 555 (2013).

[8] J. A. C. Humphrey, Fluid mechanic constraints on spider ballooning, Oecologia 73, 469 (1987).

[9] A. M. Reynolds, D. A. Bohan, and J. R. Bell, Ballooning dispersal in arthropod taxa with convergent behaviours: Dynamic properties of ballooning silk in turbulent flows, Biol. Lett. 2, 371 (2006).

[10] A. M. Reynolds, D. A. Bohan, and J. R. Bell, Ballooning dispersal in arthropod taxa: Conditions at take-off, Biol. Lett. 3, 237 (2007).

[11] L. Zhao, I. N. Panayotova, A. Chuang, K. S. Sheldon, L. Bourouiba, and L. A. Miller, Flying spiders Simulating and modeling the dynamics of ballooning, in Women in Mathematical Biology, edited by A. Layton and L. Miller, Association for Women in Mathematics Series Vol. 8 (Springer, Cham, 2017).

[12] M. Cho, P. Neubauer, C. Fahrenson, and I. Rechenberg, An observational study of ballooning in large spiders: Nanoscale multifibers enable large spiders' soaring flight, PLoS Biol. 16, e2004405 (2018).

[13] J. M. Schneider, J. Roos, Y. Lubin, and J. R. Henschel, Dispersal of Stegodyphus dumicola (Araneae, Eresidae): They do balloon after all!, J. Arachnol. 29, 114 (2001).
[14] A. Perolo, A. Castiglioni, and D. Ferri, Electrification of polymers during capillary extrusion, AIP Conf. Proc. 1914, 040007 (2017).

[15] K. Kronenberger and F. Vollrath, Spiders spinning electrically charged nano-fibres, Biol. Lett. 11, 20140813 (2015).

[16] P. W. Gorham, Ballooning spiders: The case for electrostatic flight, arXiv:1309.4731.

[17] E. L. Morley and D. Robert, Electric fields elicit ballooning in spiders, Curr. Biol. 28, 2324 (2018).

[18] C. T. R. Wilson, Atmospheric electricity, Nature (London) 68, 102 (1903).

[19] C. T. R. Wilson, Investigations on lightning discharges and on the electric field of thunderstorms, Philos. Trans. Roy. Soc. London, Ser. A 221, 73 (1921), https://www.jstor.org/stable/ 91189.

[20] M. J. Rycroft, K. A. Nicoll, K. L. Aplin, and R. G. Harrison, Recent advances in global electric circuit coupling between the space environment and the troposphere, J. Atmos. Sol. Terr. Phys. 90-91, 198 (2012).

[21] F. Barth, Spider mechanoreceptors, Curr. Opin. Neurobiol. 14, 415 (2004).

[22] D. F. McGonigle, C. W. Jackson, and J. L. Davidson, Triboelectrification of houseflies (Musca domestica L.) walking on synthetic dielectric surfaces, J. Electrost. 54, 167 (2002).

[23] M. E. Colin, D. Richard, and S. Chauzy, Measurement of electric charges carried by bees: Evidence of biological variations, J. Bioelectric. 10, 17 (1991).

[24] U. Greggers, G. Koch, V. Schmidt, A. Dürr, A. Floriou-Servou, D. Piepenbrock, M. C. Göpfert, and R. Menzel, Reception and learning of electric fields in bees, Proc. R. Soc. London B 280, 20130528 (2013).

[25] Y. Poquet, L. Bodin, M. Tchamitchian, M. Fusellier, B. Giroud, F. Lafay, A. Buleté, S. Tchamitchian, M. Cousin, M. Pélissier et al., A pragmatic approach to assess the exposure of the honey bee (Apis mellifera) when subjected to pesticide spray, PLoS ONE 9, e113728 (2014).

[26] Remcom Inc., XFdtd 3D electromagnetic simulation software, https://www.remcom.com/xfdtd-3d-em-simulation-software.

[27] R. H. Landau, M. J. Páez, and C. C. Bordeianu, Computational Physics: Problem Solving with Python, 3rd ed. (Wiley-VCH, Weinheim, Germany, 2015). 
[28] A. Penell, F. Raub, and H. Höfer, Estimating biomass from body size of European spiders based on regression models, J. Arachnol. 46, 413 (2018).

[29] E. Steven, J. G. Park, A. Paravastu, E. B. Lopes, J. S. Brooks, O. Englander, T. Siegrist, P. Kaner, and R. G. Alamo, Physical characterization of functionalized spider silk: Electronic and sensing properties, Sci. Technol. Adv. Mater. 12, 055002 (2011).

[30] R. B. Suter, Ballooning: Data from spiders in freefall indicate the importance of posture, J. Arachnol. 20, 107 (1992).

[31] H. P. Erickson, Size and shape of protein molecules at the nanometer level determined by sedimentation, gel filtration, and electron microscopy, Biol. Proceed. 11, 32 (2009).

[32] R. Gerhard-Multhaupt, W. Künstler, G. Eberle, W. Eisenmenger, and G. Yang, High space-charge densities in the bulk of fluoropolymer electrets detected with piezoelectrically generated pressure steps, in Space Charge in Solid Dielectrics, edited by J. C. Fothergill and L. A. Dissado (Dielectrics Society, Leicester, England, 1998), pp. 123-132.
[33] F. Bonacci, A. Di Michele, S. Caponi, F. Cottone, and M. Mattarelli, High charge density silica micro-electrets fabricated by electron beam, Smart Mater. Struct. 27, 075052 (2018).

[34] K. Böer, Introduction to Space Charge Effects in Semiconductors, Springer Series in Solid-State Sciences Vol. 160 (Springer, Heidelberg, 2010).

[35] F. Flores, D. Graebling, A. Allal, and C. Guerret-Piécourt, Modelization of flow electrification in a polymer melt, J. Phys. D 40, 2911 (2007).

[36] G. G. Touchard, T. W. Patzek, and C. J. Radke, A physicochemical explanation for flow electrification in low-conductivity liquids in contact with a corroding wall, IEEE Trans. Ind. Appl. 32, 1051 (1996).

[37] A. Nadler, E. Raveh, U. Yermiyahu, M. Lado, A. Nasser, M. Barak, and S. Green, Detecting water stress in trees using stem electrical conductivity measurements, Soil Sci. Soc. Am. J. 72, 1014 (2008).

[38] E. Jeon, S. Baek, S. Choi, K. S. Park, and J. Lee, Real-time monitoring of electroconductivity in plants with microscale needle probes, Environ. Control Biol. 56, 131 (2018). 